\title{
ASPECTOS DEL DERECHO A LA PROTECCION DE LA SALUD COMO GARANTIA FUNDAMENTAL DE UN ESTADO DE DERECHO
}

\author{
SERGIO ALVAREZ ROJAS \\ Profesor de Derecho Administrativo \\ Universidad de Valparaíso
}

1. Iniciación. Dentro de los Derechos Humanos cuya existencia se ha reconocido expresamente y que han significado atributos inherentes al ser humano por su condición de tal, están los Derechos Sociales, tales como el derecho al trabajo y su libre elección; a la seguridad social; a la protección a la maternidad y del menor abandonado; el Derecho a la salud; la protección del medio ambiente y otros.

De los principios que informan esta materia también se ha reconocido que la persona es el fundamento de los derechos humanos, porque El hombre constituye la raíz de estos derechos y porque representa la síntesis de todos los esfuerzos de una sociedad.

De allí que no se puede atropellar al hombre a pretexto de defender a la sociedad, porque como se ha sostenido con insistencia "el hombre no ha sido hecho para servir al Estado, sino el Estado para servir al Hombre". El Derecho a la Salud ha sido incorporado por la Carta de las Naciones Unidas, como una facultad esencial del individuo y las comunidades. "El acceso a la salud es una medida del grado de justicia social que puede existir en un país al igual que la alimentación, la educación, la vivienda, etc" $"$.

Aspectos Legales: Art. 19 №9. Constitución Política. 1980.

- Decreto Ley № 2.763 de 1979 sobre Reorganización del Ministerio de Salud y crea los Servicios de Salud.

- D. L. № 1-3063 de 1980. Permite traspaso administración múnicipal de Consultorios y Postas Rurales.

1“Salud Pública y un Proyecto Alternativo"; Publicación Colegio Médico de Chile A. G.; Volumen 39. 1987. Dr. Jorge Jiménez de la Jara. 
- D.F.L. № 3. Crea Instituciones Salud Previsional (ISAPRE). Sustituye Servicios de Salud y FONASA en otorgamiento prestaciones y beneficios de Salud.

- Ley № 18.469 de 1985. Establece igualdad en materias de sistemas de salud; elimina diferencia entre el régimen de salud y la calidad previsional; establece aporte financiero según cápacidad económica del usuario.

- D.L. 3.500. Fija cotización đe 7\% en trabajadores y pensionados. Complemen tado por Ley № 18.754 y D. L. 3.501 que nivèla la cotización al $7 \%$ en todas las Cajas de Previsión.

— Leyes № 18.566 y 18.675 , aumenta base imponible cálculo cotización previlsional sector público y permite $2 \%$ cotización adicional descontándolo de impuestos.

- Código Sanitario. Contịene normas generales sobre Protección y fomento de la salud y otorga a la autoridad la potestad sancionadora.

— Ley № 18.575 de 1986. L.O. C. de Bases Generales Administración delEstado.

2.Algunos Análisis de la Norma Constitucional. Es de todos conocido que el Sector Salud constituye un componente muy sensible de factores o parámetros por los cuales se puede medir el avance social de un conglomerado humano, de una región y/o de un país.

2.1. Para analizar seriamente los componentes de esta situación, no cabe duda que hay que establecer determinados puntos de referencia, entre los cuales no pueden faltar la Organización y Estructura Administrativa; la Infraestructura, compuesta por Establecimientos Hospitalarios, camas: índice ocupacional y promedio de días estada; Recursos Humanos, Abastecimientos y Sumunistros para la atención médica y recursos materiales; Recursos Financieros e Indicadores Económicos.

2.2. La Constitución Política de 1980 establece que la Constitución asegura a todas las personas el derecho a la protección de la salud. El Estado debe proteger el libre acceso, coordinar y controlar las Acciones de Salud: protección, fomento, recuperación, rehabilitación de la salud. Se establece también el reconocimiento de instituciones públicas o privadas y velar por el derecho de libre elección del sistema.

La salud es considerada un elemento de desarrollo, un patrimonio nacional y una responsabilidad irrenunciable del Estado.

Por lo tanto, si bien pueden crearse sistemas de atención médica privadas, ej. las ISAPRES, elEstado seguirá siendo el único responsable de la salud de la población. 
Se trata de un derecho humano, establecido en la Constitución Política y en la medida que se pueda cumplir y perfeccionar, será una garantía fundamental en un Estado de Derecho.

Este derecho se inicia en la concepción, se mantiene toda la vida y es independiente de la condición social, edad, religión o sexo. Este derecho se ejerce mediante el acceso libre e igualitario a las acciones de salud que son: fomento, protección, recuperación y rehabilitación.

La atención de salud descansa fundamentalmente en una máxima cobertura de esas acciones integradas, coordinadas y prestadas de manera oportuna, continua y eficiente.

2.3. Unà Política de Salud.

a) Política Organizativa. que debe comprender:

- El perfeccionamiento de la estructura superior del Ministerio de Salud, conforme a los arts. $20,21,22,23$, y 24 , en relación con el art. 10 de la Ley № 18.575 de Bases Generales de la Administración del Estado, en concordancia, este último, con el artículo 58 de la Ley $\mathrm{N}^{2} 18.834$ que aprobó el Estatuto Administrativo.

- El Ministerio dirige el Sector.

- Define la Doctrina y las Políticas de Salud.

- Planifica y Coordina.

- Dicta Normas.

- Controla y evalúa. Ministeriales.

A nivel regional, el Ministerio actúa a trayyés de Secretarías Generales

Dentro de este aspecto, cabría destacar la creación de los Servicios Kegionales $\mathrm{y}$. Metropolitano de Salud, Organos autónomos descentralizados y desconcentrados; con personalidad jurídica, encargados de ejecutar las acciones de salud sobre las personas y sobre el ambiente.

b) Política de desarrollo de recursos humanos, físicos, financieros, de educación y comunicaciones, implementados con una Política de investigación.

3. Financiamiento de la Salud. Un análisis especial merece este rubro. Se ha sostenido con sólidos argumentos que el financiamiento del sistema público de salud se ha caracterizado en el último deceǹio por los siguientes hechos:

a) Disminución de los ingresos totales por disminución de aporte fiscal.

b) Aumento de los aportes previsionales, lo que ha significado mayor descuento a los trabajadores.

c) Disminución del gastó global, especialmente en los rubros de inversión y personal. 
El anterior gobierno dictó disposiciones legales destinadas a favorecer el desarrollo del sector privado de Salud, al tiempo que "redujo drásticamente la inversión en el Sector Público". De allí que lo que se ha debatido es si la Salud va a ser considerada como un bien sujeto a las leyes del mercado o un servicio a la comunidad, al cual tenga acceso real toda la población ${ }^{2}$.

Al compás de la discusión no cabe duda que la disminución del gasto fiscal, impuesto autoritariamente a través de un sistema económico de la Escuela de Chicago, se fue reduciendo gravemente el financiamiento del Sector Público de Salud, hasta provocar la disminución de la atención médica hospitalaria hasta límites intolerables.

Como es sabido, la Administración Pública se organiza a través de un conjunto vertebrado de normas de Derecho Público, que establece su estructura orgánica, regula las relaciones con los particulares y con sus propios funcionarios. Estas normas tienen características especiales que modifican el Derecho Común, otorgan poderes jurídicos a los Gobemantes y colocan al particular en una situación pasiva, que se explica a través de la subordinación del interés particular al interés público, representado por el Estado.

Dentro de las obligaciones típicas de la Administración moderna, ocupa un lugar preferente la atención de la salud pública de la población, la que se consagra en disposiciones con rango constitucional, perque se ha estimado como un componente esencial del desarrollo y del nivel de vida de los pueblos.

Se ha sostenido que un Servicio de Salud es una empresa pública. Es pública porque es estatal y sirve a toda la comunidad; es empresa, porque tiene objetivos y metas técnicas que cumplir con eficacia y economía.

4. La Administración de los Servicios de Salud. La Administración Pública, en general, y los Servicios de Salud, en particular, son el medio a través del cual un Gobierno planifica, formula y realiza una política. La naturaleza y características de los procesos administrativos en el sector público, determinan la capacidad de ese Gobierno para impulsar o detener el desarrollo económico y social ${ }^{3}$.

Es importante el Derecho en la Administración de Salud y de la legislación como instrumento en el desarrollo administrativo de lọs Servicios de Salud.

No obstante, la técnica legislativa referida a los variados campos de la salud pública, ya sea en la organización; estructura y funcionamiento de los

2"Salud en Chile: el problema de fondo" Mensaje. Octubre 1979. Marżo-Abril 1986. Dr. Alejandro Goic G.

3"Administración de Salud y Derecho"; Sergio Alvarez, Eduardo Jara. Revista Ciencias Sociales. Universidad de Chile - Valparaíso- 1975. 
Servicios; así como'en la aplicación de la potestad que la Autoridad Sanitaria debe ejercer, y en general, en el resguardo de la norma jurídica frente a su órbita de acción, es insuficiente si no cuenta con la colaboración y orientación de otras técnicas, políticas y planificación, a las que lo jurídico debe prestar asesoría.

Esta es la suprema justificación de lo jurídico en el campo de la salud, o sea, su existencia por sí misma no valora ni tiene razón de existir, sino al servicio de otros procedimientos, técnicas y valoraciones puestas al servicio de la salud colectiva.

5. Funciones y Acciones de Salud. La Constitución Política, en la norma ya citada dice que el "Estado protege el libre e igualitario acceso a las acciones de promoción, protección y recuperación de la salud y de "Rehabilitación del individuo"4.

Resulta de interés establecer cuáles son estas acciones, muchas veces desconocidas para profesionales que no han estado en contacto con el Sector Salud.

A) Acciones de Promoción o. Fomento de la Salud. Ellas tienden a promover el óptimo desarrollo físico y mental del ser humano. Constituye deber fundamental del Estado, la promoción de óptimas condiciones de salud en el niño, la protección de la madre y el bienestar de la familia. En éstas encontramos las siguientes:

- Atención Materno-Infantil, a cargo de la atención extrahospitalaria de la madre y el niño y de la entrega de leche a menores de 2 años.

- Regulación de la natalidad.

- Nutrición.

- Atención al menor en situación irregular.

- Salud mental. Programas Psiquiátricos, Alcoholismo, drogas.

- Proporcionar información y servicios que promuevan el ejercicio de la paternidad responsable y una orientación adecuada hacia la vida familiar.

- Prestar atención para adoptar las medidas preventivas, de tratamiento o rehabilitación que sean procedentes para proteger a la infancia, niñez y adolescencia, en especial vulnerabilidad biológica, moral y económico social y promoverá de un modo especial el reforzamiento de la estructura familiar.

B) Acciones de Protección de Salud. Tienden a eliminar los riesgos resultantes de la vida en común. Aquí se desarrollan las siguientes funciones:

- Epidemiología: Encargada de tomar las medidas de control de las enfermedades infecciosas o transmisibles, agudas ō crónicas.

${ }^{4}$ Constitución Política de Chile. Artículo 19 № 9 
Para mayor conocimiento es del caso señalar que las enfermedades transmisibles se pueden diversificar de la siguiente manera:

1) Enfermedades que se propagan por vía respiratoria:

- Sarampión

-Rubeola

-Viruela

-Enfermedades respiratorias agudas virales

-Influenza

-Difteria

-Tos ferina

-Meningitis epidémica

-Enfermedades estreptocócicas

2) Enfermedades que se propagan por vía digestiva:

-Enfermedades diarreicas agudas

-Intoxicación alimentaria

-Tifoidea

- Hepatitis viral

-Enfermedades producidas por enterovirus

-Poliomelitis

3) Enfermedades que se propagan por contacto directo:

-Enfermedades venéreas

-Infecciones intrahospitalarias

4) Enfermédades trasmisibles de curso crónico:

-Tuberculosis

-Lepra

5) Enfermedades cuarentenables:

- Cuarentenà intervacional

- Cólera

-Fiebre amarilla

-Peste

- Tifus exantemática 
- Sanidaa Internacional.

- Higiene Ambiental. Responsable del control y promoción del saneamiento ambiental (Provisión Agua Potable, eliminación de excretas, basuras, insectos, roedores, vivienda).

- Control de Alimentos. Encargada de la supervigilancia de la calidad de los alimentos (contaminados, adulterados) y de los riesgos de contaminación que éstos pueden sufrir.

- Higiene Industrial y Medicina del Trabajo. Cuya labor consiste en la inspección de los locales de trabajo y en el diagnóstico y prevención de las enfermedades profesionales, control de la contaminación ambiental y del agua.

C) Recuperación de la Salud. Proveen los medios para actuar una vez producida la enfermedad.

- Atención médica del enfermo

- Responsable del funcionamiento de hospitales y consultorios. Construcción hospitalaria

- Odontología

- Farmacia

- Servicio Social

D) Rehabilitación. Esta acción involucra todos los procesos que llevan a la recuperación de una función de salud que se estima perdida (secuelas). Ellas pueden ser psiquiátricas, físicas, pedagógicas, de lenguaje, etc.

6. La Legislación de Salud. Esta es uno de los instrumentos fundamentales para el desarrollo eficaż de los Servicios, puesto que sin su apoyo no pueden llevarse a cabo de manera satisfactoria las acciones de salud, tales como: prevención de enfermedades; fomento de la salud; protección del medio ambiente; la administración de hospitales y otros aspectos referidos al desarrollo administrativo de las Instituciones de Salud.

Los objetivos principales de esta Legislación de Salud, ciertamente especializada, son, entre otros, definir la estructura y los poderes de los organismos encargados de la administración de salud en todos sus niveles; definir los derechos y obligaciones y prescribir las normas y condiciones que rigen las materias específicas a las cuales se aplica la legislación sanitaria en esta materia y, finalmente, establecer los derechos y obligaciones de los Servicios de Salud, respecto de sus funcionarios. Dicho de otra manera, el instrumento legal dentro del 
quehacer de la órbita jurídica, pone a disposición de los técnicos o administradores de salud, los elementos básicos para la correcta percepción y resolución de los problemas que debe atender.

Las principales materias 'referente a lás acciones de promoción y fomento las encontramos en el Código Sanitario y en un número considerable de reglamentos complementarios del Código ${ }^{5}$.

7. Sobre el Traspaso de Postas y Consultorios de Salud a las Municipalidades.

El D.L. № 3063, de 1979, establece que es obligación de las Municipalidades "destinar preferentemente los recursos del Fondo común municipal a crear, mantener y prestar servicios a la comunidad local. 'Además, podrán tomar a su cargo servicios que estén siendo atendidos por Organismos del Sector Público o Privado"6.

El D.F.L. 1-3063, reglamenta la aplicación del anterior D.L.. Una de sus normas importantes es la que señala que este reglamento contiene normas generales por las cuales se rige la inversión de los recursos y el traspaso de los servicios del Sector Público y su consecuente trasferencia de activos, recursos financieros, recursos humanos y normas de administración financiera. El traspaso de los servicios podrán tener el carácter de provisorios o definitivos ${ }^{7}$.

Otro aspecto importante es aquel que establece que las Múnicipalidades que toman a su cargo la atención de un Servicio se ajustarán en su gestión a todas las disposiciones que sobre el particular rigen para tal actividad y estará sujeta - a la supervigilancia técnica y fiscalización que disponga la ley, de parte de las entidades y servicios especializados.

En este caso que analizamos se trata de un principio de organización administrativa, por medio del cual existe una delegación de facultades de parte del Organo que tenía tales atribuciones de una manera, a veces, excluyente. De tal forma que se pueden repartir periféricamente las competencias entre los órganos superiores y los inferiores, de tal manera que estos últimos adquieren competencias propias y exclusivas ${ }^{8}$.

El problema que surge con respecto a las Municipalidades, es que el actual sistema delega facultades en autoridades unipersonales, estrictamente de-

${ }^{5}$ Ley No 18.575, de 1986. L.O.C. de Bases Generales Administración Pública ${ }^{6}$ D.L. № 3.063. 1979.

${ }^{7}$ D.F.L. 1-3063. Reglamenta el anterior.

8"Descentralización Administrativa dentro del marco de Regionalización"; Sergịo Alvarez. VII Jomadas de Derecho Público. 1979. Universidad Católica de Valparaíso. 
pendientes del Poder Ejecutivo. A esto se agrega un cúmulo de aspectos negativos tales como capacidad técnica-administrativa, de financiamiento futuro de tales Organismos y de una planificación razonable que se ajuste a una línea de experiencia práctica que no tienen, principalmente por inestabilidad funcionaria partiendo del Jefe Municipal, que es una persona de confianza del Ejecutivo y por no haber una carrera funcionaria, estable e idónea.

Lo concreto es que, si bien bajo una apreciación de Derecho Administrativo y Constitucional la idea de descentralización no es desechable, estimamos que en la actualidad pueden significar mayores dificultades en un manejo tan complejo como son las acciones de salud, tanto preventivas como de fomento, aun cuando estos Establecimientos traspasados sólo puedan atender una patología de enfermedades que se derivan a la atención hospitalaria.

Este proceso del traspaso de postas y consultorios rurales de salud se ha completado regularmente alrededor del año 1986. Son 1.200 establecimientos en total, ya que se incluyen algunos consultorios generalmente urbanos, especialmente en Santiago.

- Todo lo anterior representan aspectos de la Atención Primaria de Salud, y para superar deficiencias se requiere acciones de emergencia en las que se incluyen:

a) La extensión de horarios y el aumento de la capacidad de diagnóstico y tratamiento de estos Consultorios;

b) Implementar Consultorios Periférićos de Urgencia Ambulatoria;

c) Subsanar el desabastecimiento de insumos, las malas condiciones de atención a los pacientes y de trabajo para los profesionales médicos y personal que trabaja en ellos.

\section{Algo sobre las ISAPRES.}

Estas Instituciones nacieron en 1982, dentro del gobierno anterior, a través de - D.F.L. N2 3. Estas Instituciones de Salud Previsional, han intentado captar las cotizaciones de salud y sustituyen a los Servicios de Salud FONASA, en el otorgamiento de prestaciones y servicios, aun cuando permite los convenios con los Servicios de Salud en la atención de pensionados, en Unidades de Cuidados Intensivos de Hospitales y ên Servicios de Urgencia de éstos.

Según las opiniones más optimistas el sistema de ISAPRES, es elocuente consignar que en apenas ocho años y sobre la base de una total afiliación, las 33 Instituciones hoy existentes han llegado a cubrir las necesidades de 2.200.000, personas. Otro dato interesante es el que sostienen los dirigentes de estas Instituciones privadas, con fines de lucro, al expresar que el promedio de sus afiliados 
al sistema se ha ido disminuyendo paulatinamente "de tal modo que hoy el $66 \%$ de los cotizantes del sistema privado percibe un ingreso mensual de menos de $\$ 120.000$.- y la mitad de estos últimos es inferior a $\$ 60.000$.-. Agrega que ajustándose con mucha flexibilidad a las necesidades y posibilidades de dichos cotizantes, el sistema de ISAPRES ofrece actualmente un gran número de planes de salud, que supera ya los 400 y que cubre una creciente gama de rubros".

Este sistema se funda en realidad en el derecho de la persona a la protección de salud; en segundo término, acceso libre, igualitario y garantizado por el Estado a las prestaciones de Salud; en el rol subsidiario del Estado frente al problema de la salud donde con quien atenderse, incluyendo expresamente las estructuras del sector privado.

Con todo lo anterior, podemos sostener que el público, en general, tiene imágenes cóntradictorias de ellas, producto de la gran propaganda publicitaria, y las frustraciones evidentes que algunas de ellas producen en el proceso de cumplimiento de sus promesas de ventas. Existen ISAPRES de muy variada naturaleza y la tendencia es que estas diferencias se acentúan. La creación de nuevas ISAPRES gestionadas por Cajas de Compensación, los bienestares de las empresás y los trabajadores mismos dará una contradicción aumentada a este tipo de seguros de salud.

Recientemente, en Noviembre de 1990, se ha sostenido por la Superintendencia de ISAPRES que se han detectado "problemas de desinformación y falta de trasparencia de los contratos". La desinformación de los usuarios limita sus derechos y perjudica la posibilidad de comparación. Queda poca claridad en los contratos, puesto que no estipulan adecuadamente sus derechos y obligaciones: existen aranceles diferenciales muy discímiles; hay excesivas restriccionès al uso de los beneficios del sistema. En estos últimos casos, se encuentran los pensionados, los pacientes afectados, por enfermedades mentales y por cáncer que no incluyen la mayoría de las ISAPRES en sus programas. $E_{j}$. cuando los pacientes jubilan son referidos automáticamente al sistema estatal.

Para finalizar estos comentarios sobre las ISAPRES, debemos sostener que si bien, estas Instituciones fueron motivo de una reforma impuesta por la vía del Decreto con Fuerza de Ley, se debe reconocer su legitimidad y su existencia, a pesar, que como se ha expresado, se unieron muchas características de discriminación sobre tipos de personas, condiciones patológicas, todo lo cual ha significado que su presencia esté discutida en el sistema de Salud.

Por otra parte, se debe reconocer que dichas Instituciones han permitido que exista una posibilidad de ahorro para la salud y que organice un subsistema que hoy cubre al $12 \%$ de la población y cuyos rẹcursos han estimulado efectivamente

"III Seminario Coyuntura de Salud"; 1989. Asociación de Isapres A. G. 
al sector privado de atención médica, perfeccionando sus infraestructuras y modernización tecnológica.

Sin embargo, cabe tener presente, que su orientación general las lleva a "una acción curativa dejando de lado los aspectos de promoción y de prevención de salud", aparte de las otras críticas que se han formulado.

\section{CONCLUSIONES}

La intención de desarrollar este tema se ha basado en las siguientes razones:

1) Porque estamos convencidos que la Protección del Derecho a la Salud, que el Estado se obliga a garantizar, supone el ejercicio real de asegurar el acceso a una atención de salud digna y eficiente y establece las condiciones adecuadas de Salud Pública para toda la población.

2) Estimamos que, aún considerando esta obligatoriedad del Estado, la responsabilidad en su cumplimiento es y debe ser compartido solidariamente por la Comunidad y el individuo, para mejorar las condiciones de Salud.

3) Estimamos que la Salud es un supremo bien consubtancial a la vida de las personas, que implica valores superiores como respeto, dignidad, solidaridad e igualdad de oportunidades.

4) Exponer para un mayor conocimiento de los especialistas en Derecho Constitucional y Administrativo las materias que comprenden las "acciones sobre Promoción y/o Fomento, Protección, Recuperación y Rehabilitación del individuo", que señala el artículo 19 №.9, sobre los Derechos y Deberes, que la Constitución asegura a todas las personas, conceptos sobre los que muchas veces no hay claridad.

5) Dar una somera apreciación, de la importancia del Derecho Administrativo, en cuanto dice relación con la Organización, Estructura y Funciones de un Servicio de Salud.

6) Finalmente, expresar, a modo general, una opinión sobre dos temas controvertidos, que caen dentro de la órbita de lo jurídico-administrativo, como es el traspaso a las Municipalidades de Consultorios y Postas de Salud y respecto a las Instituciones de Salud Previsional (ISAPRES), cuyas prestaciones y funcionamiento han sido muy controvertidas. 